\title{
Comparative Genomics of Potato Common Scab-Causing Streptomyces spp. Displaying Varying Virulence
}

\author{
Cindy Hudec ${ }^{1}$, Adrien Biessy ${ }^{2}$, Amy Novinscak ${ }^{3}$, Renée St-Onge ${ }^{1}$, Simon Lamarre', \\ Jochen Blom ${ }^{4}$ and Martin Filion ${ }^{2 *}$
}

${ }^{1}$ Department of Biology, Université de Moncton, Moncton, NB, Canada, ${ }^{2}$ Saint-Jean-sur-Richelieu Research and Development Centre, Agriculture and Agri-Food Canada, Saint-Jean-sur-Richelieu, QC, Canada, ${ }^{3}$ Agassiz Research and Development Centre, Agriculture and Agri-Food Canada, Agassiz, BC, Canada, ${ }^{4}$ Bioinformatics and Systems Biology, Justus-Liebig-Universität Giessen, Giessen, Germany

OPEN ACCESS

Edited by:

Feng Gao,

Tianjin University, China

Reviewed by:

Bart Devreese

Ghent University, Belgium Jose Carlos Huguet-Tapia,

University of Florida, United States

${ }^{*}$ Correspondence: Martin Filion

martin.filion@canada.ca

Specialty section:

This article was submitted to Evolutionary and Genomic Microbiology, a section of the journal Frontiers in Microbiology

Received: 28 May 2021

Accepted: 13 July 2021 Published: 03 August 2021

Citation: Hudec C, Biessy A, Novinscak A, St-Onge R, Lamarre S, Blom J and

Filion M (2021) Comparative

Genomics of Potato Common Scab-Causing Streptomyces spp.

Displaying Varying Virulence.

Front. Microbiol. 12:716522.

doi: 10.3389/fmicb.2021.716522
Common scab of potato causes important economic losses worldwide following the development of necrotic lesions on tubers. In this study, the genomes of 14 prevalent scab-causing Streptomyces spp. isolated from Prince Edward Island, one of the most important Canadian potato production areas, were sequenced and annotated. Their phylogenomic affiliation was determined, their pan-genome was characterized, and pathogenic determinants involved in their virulence, ranging from weak to aggressive, were compared. 13 out of 14 strains clustered with Streptomyces scabiei, while the last strain clustered with Streptomyces acidiscabies. The toxicogenic and colonization genomic regions were compared, and while some atypical gene organizations were observed, no clear correlation with virulence was observed. The production of the phytotoxin thaxtomin A was also quantified and again, contrary to previous reports in the literature, no clear correlation was found between the amount of thaxtomin A secreted, and the virulence observed. Although no significant differences were observed when comparing the presence/absence of the main virulence factors among the strains of S. scabiei, a distinct profile was observed for S. acidiscabies. Several mutations predicted to affect the functionality of some virulence factors were identified, including one in the bldA gene that correlates with the absence of thaxtomin A production despite the presence of the corresponding biosynthetic gene cluster in S. scabiei LBUM 1485. These novel findings obtained using a large number of scab-causing Streptomyces strains are challenging some assumptions made so far on Streptomyces' virulence and suggest that other factors, yet to be characterized, are also key contributors.

Keywords: common scab, Streptomyces, genomics, pathogenicity island, virulence, thaxtomin

Abbreviations: AA, amino acid; ANI, average nucleotide identity; att, attachment site; BLAST, Basic Local Alignment Search Tool; $c b s$, CebR-binding site; CDSs, coding DNA sequences; CFA-Ile, N-coronafacoyl-L-isoleucine; CIME, cis-mobilizable element; CK, cytokinin; CS, common scab; CR, colonization region; is DDH, in silico DNA-DNA hybridization; GGDC, genome to genome distance calculator; HGT, horizontal gene transfer; IAA, Indole-3-Acetic Acid; ICE, integrative and conjugative elements; LC-MS, liquid chromatography coupled - mass spectrometry; MAFFT, Multiple Alignment using Fast Fourier Transform; PAI, pathogenicity island; PEI, Prince Edward Island; SNP, single nucleotide polymorphism; PROVEAN, Protein Variation Effect Analyzer; TR, toxicogenic region; TSB, tryptic soya broth; VFs, virulence factors; VFDB, Virulence Factor Database. 


\section{INTRODUCTION}

Potato plants are subjected to various stresses, including diseases caused by numerous bacterial, oomycete and fungal plant pathogens, affecting yield and market values (Stevenson et al., 2001). Common scab (CS) of potato is highly prevalent in Prince Edward Island (PEI), which is one of the most important potatoproducing Canadian provinces (Statistics Canada, 2021). CS causes necrotic lesions on the tuber surface, which affect potato transformation and commercialization, resulting in economic losses estimated between 15.3 and 17.3 million dollars per year in Canada (Hill and Lazarovits, 2005). This worldwide disease is caused by a few closely related Streptomyces species (Braun et al., 2017). Among the dozen Streptomyces species causing CS reported so far, the best characterized are Streptomyces scabiei, Streptomyces acidiscabies, and Streptomyces turgidiscabies (Loria et al., 2006).

Advances in next-generation sequencing technologies have resulted in an upsurge of bacterial genome sequences available. However, so far only few plant pathogenic Streptomyces spp. genomes have been sequenced (18 S. scabiei; ten S. acidiscabies, and two S. turgidiscabies, NCBI May 2021). Although these genomic sequences have provided new opportunities to perform comparative genomic studies, additional genomes will be required to perform large-scale analyses. Comparisons between pathogenic and saprophyte Streptomyces spp. have revealed numerous genes and gene clusters that contribute to pathogenicity, virulence and/or plant-microbe interactions (i.e., pathogenome) (Bignell et al., 2010). Moreover, functional analyses of some of these genetic determinants have confirmed their implication in the development of CS symptoms (Bignell et al., 2010).

The main pathogenicity determinant in scab-causing Streptomyces spp. has been identified as a cyclic dipeptide $(2,5-$ diketopiperazine) phytotoxin called thaxtomin A, which inhibits cellulose biosynthesis on actively growing tubers (Lawrence et al., 1990; King et al., 1991; Bignell et al., 2010). A positive correlation between the severity of the symptoms observed (virulence) and thaxtomin A production has also been reported (Kinkel et al., 1998). Genes involved in thaxtomin A biosynthesis, consisting of a cluster of six genes: $t x t A, t x t B, t x t C, t x t D, t x t E$, and $t x t H$ (Bignell et al., 2014b), are localized in a cis-mobilizable element (CIME) in a toxicogenic region (TR1, $20 \mathrm{~kb}$ ) (Zhang and Loria, 2017). This region is highly conserved among thaxtominproducing Streptomyces spp. and is separated from a TR2 region by an internal attachment site (att) (Chapleau et al., 2016). TR2 $(157 \mathrm{~kb})$ contains putative integrative and conjugative elements (ICE) required for mobilization and is only conserved among S. scabiei strains (Zhang et al., 2016).

Biosynthesis of thaxtomin $\mathrm{A}$ is under the strict transcriptional control of the TxtR protein, a member of the AraC/XylS family, which promotes the transcription of $t x t A, t x t B, t x t C$, and $t x t H$ (Johnson et al., 2007; Joshi et al., 2007). More specifically, cellobiose and cellotriose, which derive from cellulose present in the plant cell wall, are the main inducers of thaxtomin A production (Johnson et al., 2007). The ability of pathogenic Streptomyces to participate in the degradation of plant polymers like cellulose or suberin, have been demonstrated through the secretion of glycosyl hydrolases and esterases such as suberinase and cutinase (Komeil et al., 2013; PadillaReynaud et al., 2015; Huguet-Tapia et al., 2016). These two oligosaccharides are specifically transported inside S. scabiei by the CebEFG/MsiK transmembrane transporter (Jourdan et al., 2016). Once internalized, they interact with CebR, a transcriptional repressor of the thaxtomin A biosynthetic gene cluster. In the absence of these oligosaccharides, CebR binds to DNA via two CebR-binding sites ( $c b s ; c b s^{t x t B}$ and $c b s^{t x t A-R}$ ) localized inside $t x t B$ and between the $t x t A$ and $t x t R$ genes, repressing the expression of these txt genes. When cellobiose and cellotriose interact with CebR, the repression is lifted and thaxtomin A production begins (Francis et al., 2015). This production is also under the control of several bld genes (bldA, bldC, bldD, bldG, and bldH) principally known to participate in the morphological development and/or the production of antibiotics by Streptomyces spp. (McCormick and Flärdh, 2012; Bignell et al., 2014a).

In addition to thaxtomin $\mathrm{A}$, and notably due to the existence of pathogenic Streptomyces spp. that do not produce this toxin (Park et al., 2003; Wanner, 2004; Cao et al., 2012; Fyans et al., 2016; Lapaz et al., 2017), others phytotoxins and/or secreted proteins produced by pathogenic Streptomyces spp. have been described or are suspected to play a role in the development and/or the severity of the disease. Among them, the production of coronafacoyl (N-coronafacoyl-L-isoleucine; CFAIle) (Fyans et al., 2015; Bignell et al., 2018) and concanamycin A and B (Natsume et al., 1996, 1998; Fyans et al., 2016) have been reported in S. scabiei. Their respective biosynthetic gene clusters have never been found in other Streptomyces species causing CS. As for thaxtomin A, some bld genes have also been identified as transcriptional regulators of its production (Bignell et al., 2014a). Even if their role in CS development remains unclear, it has been demonstrated that they are nonessential pathogenicity factors but still significantly contribute to the severity of the disease as virulence factors (VFs). On potato tuber tissues, CFA-Ile has been shown to increase necrosis and pitting (Cheng et al., 2019), and concanamycin A and $B$, which have a synergic effect with thaxtomin $A$, have also been shown to contribute to the formation of pitted lesions (Natsume et al., 2017).

Recent studies have revealed the production of additional phytotoxins by Streptomyces spp. also impacting lesion types and/or disease severity. Streptomyces sp. strain GK18 isolated from a scab lesion in Iran was shown to produce borrelidin, a macrolide causing deep and black holes on potato tuber slices (Cao et al., 2012). A strain of Streptomyces niveiscabiei (strain ST1015) isolated in Uruguay was shown to produce desmethylmensacarcin, a polyketide more aggressive than thaxtomin A that also causes deep necrotic lesions on potato tubers (Lapaz et al., 2019). Several phytopathogenic Streptomyces strains isolated in Japan produce macrolide FD-891, which induces necrosis on potato tuber slices (Natsume et al., 2005). Natsume et al. (2018), have also isolated a S. turgidiscabies strain in Sweden producing fridamycin $\mathrm{E}$, which reduces or inhibits the sprouting of potato microtubers (Natsume et al., 2018). 
In addition to the phytotoxins secreted by scab-causing Streptomyces spp., these pathogens produce phytohormones which facilitate the infection of potato plants, notably by altering the plant hormonal signaling pathways. Kers et al. (2004) identified a gene cluster in S. turgidiscabies highly similar to the plant fasciation (fas) operon present in Rhodococcus fascians. In this species, the fas operon is required for pathogenicity and allows the biosynthesis of cytokinin (CK), a plant growth hormone (Eason et al., 1996). CK production by S. turgidiscabies and its involvement in the pathogenicity was then confirmed by Joshi and Loria (2007). The production of auxin (indole-3-acetic acid, IAA) in S. scabiei was also demonstrated (Bignell et al., 2010) and its contribution to virulence was observed (Hsu, 2010; Legault et al., 2011). A homolog of a gene encoding an ethyleneforming enzyme (efe) that catalyzes ethylene production (Bignell et al., 2010), which could be involved in the colonization of the pathogen in planta (Weingart et al., 2001), was also described in S. scabiei.

Finally, in addition to these phytotoxins and phytohormones, scab causing Streptomyces spp. are also able to secrete proteins (effectors or extracellular enzymes) which contribute to virulence. For example, in S. scabiei, scabin belonging to the mono-ADPribosyltransferase family, was identified as a VF (Lyons et al., 2016). The nec1 and tom $A$ genes, which encode, respectively, a necrotic protein and a putative tomatinase probably involved in the suppression of plant defense responses have also been reported in some pathogenic Streptomyces spp. as VFs (Loria et al., 2006). Both of these genes are located in a chromosomal region of $105 \mathrm{~kb}$ conserved among Streptomyces species, the colonization region (CR) (Lerat et al., 2009; Zhang et al., 2016). In $S$. turgidiscabies, this region and the TR region belong to a mobile pathogenicity island (PAI) that can be horizontally transferred from pathogenic to saprophytic Streptomyces species, resulting in the emergence of new pathogenic phenotypes (Kers et al., 2004; Zhang et al., 2016). Despite its polyphyletic origin, S. scabiei appears to be the oldest common scab-causing pathogen that has participated to the emergence of others pathogenic species such as S. acidiscabies and S. turgidiscabies, through lateral gene transfer (Chapleau et al., 2016; Huguet-Tapia et al., 2016).

In a recent study performed in our laboratory, a collection of close to 300 prevalent scab-causing Streptomyces strains were isolated from PEI, Canada and grouped into fourteen distinct genetic groups of Streptomyces spp. (Hudec et al., 2021). Thirteen of them were taxonomically affiliated with $S$. scabiei, while the last one was affiliated with $S$. acidiscabies. Moreover, virulence assays showed strong differences in virulence, ranging them from weak (producing less than $5 \%$ of CS coverage on tubers) to aggressive strains ( $>50 \%$ tuber coverage) (Hudec et al., 2021). In order to elucidate why such unexpected variability in virulence was observed, the genomes of these 14 scabcausing genetic groups of Streptomyces spp. were sequenced and compared. First (i), pan-genome description and phylogenetic relationships between the fourteen strains were investigated to study putative links existing between virulence and general strain affiliations. Secondly, (ii) toxicogenic and colonization regions containing several pathogenic determinants were compared. Thirdly, (iii) thaxtomin A production was quantified using standardized assays to determine its implication in virulence, and finally (iv) genes involved directly or indirectly in the biosynthesis and regulation of thaxtomin A production and others known virulence determinants were compared to identify potential genetic mutations altering thaxtomin A production and/or virulence. The analyses performed in this study provide up-to-date information on the genomic diversity of pathogenic Streptomyces spp. found under commercial potato production conditions in Eastern Canada as well as its implication in virulence. A better characterization of the determinants contributing to the virulence in pathogenic Streptomyces strains is required for the development of adapted management tools under field conditions.

\section{MATERIALS AND METHODS}

\section{Bacterial Strains, Culture Conditions and Conservation}

The 14 pathogenic Streptomyces strains used in this study were previously isolated from potato tubers (cv. Prospect) harvested in PEI, Canada (Hudec et al., 2021). Originally named Streptomyces spp. G01-G14 (genetic groups 1-14), these strains were renamed Streptomyces scabiei LBUM 1475, LBUM 1477, LBUM 1487, LBUM 1480, LBUM 1485, LBUM 1482, LBUM 1486, LBUM 1484, LBUM 1483, LBUM 1488, LBUM 1481, LBUM 1478, LBUM 1479 , and Streptomyces acidiscabies LBUM 1476, respectively. The strains were grown in the dark at $28^{\circ} \mathrm{C}$ under continuous shaking (120 rpm) for 4 days using $100 \mathrm{ml}$ of tryptic soya broth (TSB, BD Biosciences, Franklin, NJ, United States), and were conserved at $-80^{\circ} \mathrm{C}$ in TSB supplemented with $50 \%$ glycerol (vol/vol).

\section{Genome Sequencing, Assembly and Annotation}

Genomic DNA was extracted using the DNeasy UltraClean Microbial Kit (Qiagen, Mississauga, ON, Canada) as previously described (Hudec et al., 2021) and purified using Agencourt AMPure XP beads (Beckman Coulter, Mississauga, ON, Canada) according to the manufacturer's instructions.

Genomes were sequenced using Single Molecule, Real-Time sequencing technology on a PacBio RS II sequencer (Pacific Biosciences, Menlo Park, CA, United States) and assembled using the HGAP pipeline (Chin et al., 2013) at the McGill University and Genome Quebec Innovation Centre located in Montreal, QC, Canada.

The genome sequences obtained in this study were annotated using the Prokaryotic Genome Annotation Pipeline (PGAP) (Tatusova et al., 2016) and were deposited in DDBJ/ENA/GenBank under the accession numbers provided in Table 1. The versions described in this paper are the first versions.

\section{Thaxtomin A Quantification Using LC-MS}

Streptomyces strains were first cultured in TSB as previously described. Standardization of the bacterial quantities, culture conditions in oat bran broth media and collection of the supernatants were performed as described by Jiang et al. (2018). 
Solid phase extraction cartridges (Bond-Elut-C18, $100 \mathrm{mg}, 1 \mathrm{ml}$, Agilent Inc., Mississauga, ON, Canada) were used to extract thaxtomin A. These columns were first preactivated with $1 \mathrm{ml}$ of $100 \%$ methanol $(\mathrm{MeOH})$ and washed with an equal volume of water. Then, $1 \mathrm{ml}$ of sample was passed through the preactivated cartridge followed by $1 \mathrm{ml}$ of water and $1 \mathrm{ml}$ of $25 \% \mathrm{MeOH}$ in water. Thaxtomin A was then eluted using $0.25 \mathrm{ml}$ of $100 \% \mathrm{MeOH}$. The eluate was combined with one volume of water with $0.1 \%$ formic acid (solvent A) immediately before being analyzed using liquid chromatography with mass spectrometry detection (LC-MS). The chromatographic separation was performed using a reverse phase HPLC column (Synergi Hydro-RP, $100 \mathrm{~mm} \times 2 \mathrm{~mm}$, Phenomenex Inc., Torrance, CA, United States). The LC conditions included an injection volume of $5 \mu \mathrm{l}$ into a column at $37^{\circ} \mathrm{C}$ eluted with a linear gradient of $0-100 \%$ solvent $\mathrm{B}$ (methanol:acetonitrile $50: 50$ ) over $10 \mathrm{~min}$ at a flow rate of $0.5 \mathrm{ml} / \mathrm{min}$. The column was further cleaned with $100 \%$ solvent B for $3 \mathrm{~min}$ and then reequilibrated with solvent $\mathrm{A}$ for $10 \mathrm{~s}$. Isolated thaxtomin $\mathrm{A}$ was detected in time-of-flight MS. The ion source was a dual electrospray (ESI) operated on positive mode. The MS conditions were as follows: gas temperature, $325^{\circ} \mathrm{C}$; capillary voltage, $3500 \mathrm{~V}$; fragmentor, $175 \mathrm{~V}$; and skimmer, $65 \mathrm{~V}$. One MS spectra per second was acquired under a mass range of $\mathrm{m} / \mathrm{z} 50-1,700$. Under these conditions thaxtomin A was eluted at $7.39 \mathrm{~min}$ with $\mathrm{m} / \mathrm{z}$ 439.1612. The concentrations of thaxtomin A were extrapolated from a standard curve of pure thaxtomin A from 0 to $2 \mu \mathrm{g} / \mathrm{ml}$ (Sigma-Aldrich Canada Co., Oakville, ON, Canada). In cases where the sample concentration was higher than $2 \mu \mathrm{g} / \mathrm{ml}$, the samples were further diluted using $0.1 \%$ formic acid in water. The chromatographic system used was an Agilent 1100 and the MS system was an Agilent 6230 (Agilent Inc.). Data acquisition was performed using the MassHunter Version B.08.00 software. The experiment set-up consisted of three independent biological replicates. Statistical analyses were performed using RStudio version 1.2.5001 (Boston, MA, United States). The R function "kruskal" with a Benjamini-Hochberg $(\mathrm{BH})$ correction, from the "Agricolae" package version 1.2-8 was used $(p<0.05)$ to compare the thaxtomin A produced among the different strains (De Mendiburu, 2019). A correlation between the amount of thaxtomin A produced in vitro and the common scab coverage observed in planta as published in a previous study (Hudec et al., 2021) (i.e., virulence) was performed using the Kendall method (De Mendiburu, 2019).

\section{Taxonomy and Phylogeny}

To determine the species affiliation of the 14 strains under study, two analyses were performed using the type strains S. scabiei NRRL B-16523 ${ }^{T}$ and S. acidiscabies NRRL B$16524^{T}$. The first one was performed using in silico DNADNA hybridization (isDDH) with Genome-to-Genome Distance Calculator - Formula 2 (GGDC 2.1) on the web-based DSMZ service available at http://ggdc.dsmz.de (Meier-Kolthoff et al., 2013). A cut-off of $70 \%$ for species affiliation was used. The second one was based on the Average Nucleotide Identity (ANI) and was calculated using the algorithm developed by Goris et al. (2007), with a 95\% cut-off for species boundary (Richter and Rosselló-Móra, 2009). We report here the averages of the reciprocal comparisons obtained on http://enve-omics.ce.gatech. edu/ani/ (Rodriguez-R and Konstantinidis, 2016). Moreover, a phylogenomic tree of the 14 Streptomyces strains under study, as well as eight additional well characterized pathogenic Streptomyces species was built using EDGAR 3.0 (Dieckmann et al., 2021). Streptomyces griseolus NRRL B-2925 ${ }^{T}$, a nonpathogenic Streptomyces species was used as an outgroup. The robustness of the inferred tree was evaluated with a bootstrap resampling method with 1000 replicates.

\section{Pan-Genome}

Coding DNA sequences (CDSs) from each genome were compared against each other to identify orthologous CDSs that are (i) present in every strain (core-genome), (ii) species- or

TABLE 1 | General genomic information on the 14 scab-causing Streptomyces spp. under study.

\begin{tabular}{|c|c|c|c|c|c|c|c|c|}
\hline Species & Strains & $\begin{array}{l}\text { Genome } \\
\text { coverage }\end{array}$ & Contig no. & $\begin{array}{c}\text { Contig } \\
\text { N50 } \\
\text { (Mb) }\end{array}$ & $\begin{array}{c}\text { Genome size } \\
\text { (Mb) }\end{array}$ & $\begin{array}{c}G+C \text { content } \\
(\%)\end{array}$ & $\begin{array}{l}\text { CDSs no. } \\
\text { (total) }\end{array}$ & $\begin{array}{l}\text { Accession } \\
\text { numbers }\end{array}$ \\
\hline S. scabiei & LBUM 1475 & $159 x$ & 1 & 9.74 & 9.74 & 71.6 & 8455 & СР043958 \\
\hline S. acidiscabies & LBUM 1476 & $140 x$ & 13 & 10.35 & 10.94 & 70.6 & 9751 & WWSX00000000 \\
\hline S. scabiei & LBUM 1477 & $148 x$ & 5 & 9.57 & 9.77 & 71.6 & 8528 & WWSW00000000 \\
\hline S. scabiei & LBUM 1478 & $63 x$ & 116 & 6.03 & 10.15 & 71.3 & 9004 & VWSV00000000 \\
\hline S. scabiei & LBUM 1479 & $78 x$ & 3 & 9.62 & 10.19 & 71.5 & 8911 & WWSU00000000 \\
\hline S. scabiei & LBUM 1480 & $153 x$ & 1 & 9.98 & 9.98 & 71.5 & 8654 & СР043957 \\
\hline S. scabiei & LBUM 1481 & $112 x$ & 5 & 9.52 & 9.87 & 71.5 & 8569 & WWST0000000 \\
\hline S. scabiei & LBUM 1482 & $167 x$ & 1 & 9.78 & 9.78 & 71.6 & 8518 & CP043956 \\
\hline S. scabiei & LBUM 1483 & $148 x$ & 3 & 9.68 & 9.82 & 71.5 & 8548 & WWSS00000000 \\
\hline S. scabiei & LBUM 1484 & $100 x$ & 2 & 9.59 & 9.63 & 71.6 & 8373 & WWSR00000000 \\
\hline S. scabiei & LBUM 1485 & $58 x$ & 58 & 6.76 & 9.87 & 71.5 & 8616 & VWSQ00000000 \\
\hline S. scabiei & LBUM 1486 & $127 x$ & 3 & 9.88 & 10.05 & 71.5 & 8803 & WWSP00000000 \\
\hline S. scabiei & LBUM 1487 & $147 x$ & 2 & 9.79 & 9.97 & 71.5 & 8695 & VWSO00000000 \\
\hline S. scabiei & LBUM 1488 & $169 x$ & 2 & 9.94 & 9.98 & 71.5 & 8630 & WWSN00000000 \\
\hline
\end{tabular}




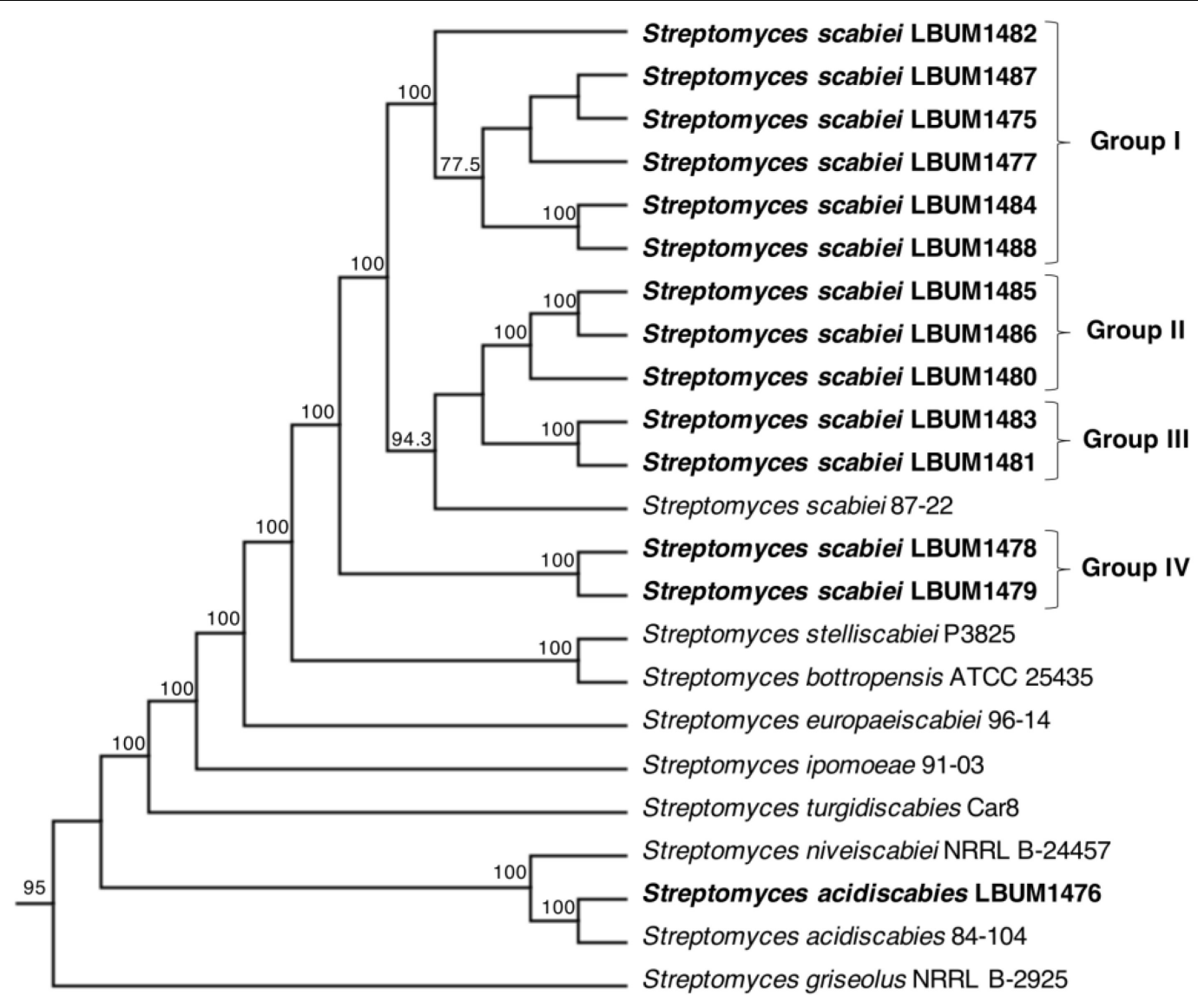

FIGURE 1 | Neighbor-joining phylogeny tree of 14 Streptomyces strains used in this study (bold) and eight phytopathogenic Streptomyces spp. reference strains. S. griseolus NRRL B-2925 was used as an outgroup. The Neighbor-Joining tree is based on the alignment of the core-genomes of these organisms (corresponding to 1577 CDSs per genome). Four S. scabiei groups were distinguished. Only bootstrap values higher than 60\% are displayed. Branches are presented as a cladogram for improved clarity.

group-specific, or (iii) unique to a given strain (singletons) using EDGAR 3.0. Orthologs were identified using bidirectional best BLAST hits using a Score Ratio Value of 0.31 as the orthology cutoff.

In order to provide information on the specific functions of the CDSs belonging to the pan-genome of these 14 strains, the protein sequences were annotated against the Clusters of Orthologous Groups database (COGs; Tatusov et al., 2000) by BLASTP search $\left(e\right.$-value $\left.<1 \times 10^{-3}\right)$ with eggNOG-mapper v2 databases (Huerta-Cepas et al., 2017) and against the Virulence Factor Database (VFDB; $e$-value $<10^{-5}$ ) (Liu et al., 2019).

\section{Analysis of the Genetic Determinants Involved in Pathogenicity and Virulence}

Based on the literature, known gene sequences related to Streptomyces' pathogenicity and virulence (Supplementary Table 3) were retrieved from the genomes of the 14 Streptomyces strains using BLASTn. The toxicogenic and colonization regions were also identified. Multiple sequence alignments (MAFFT) of these genes and gene regions were performed (Katoh and Standley, 2013) and Neighbor-Joining consensus trees based on the Jukes-Cantor distance were constructed (Saitou and Nei, 1987). To evaluate the robustness of the inferred trees, the bootstrap resampling method was used with 1000 replicates. These analyses were performed on Geneious Prime 2019.2.3 (Biomatters, Ltd., Auckland, New Zealand).

All nucleotide sequences of the $13 \mathrm{~S}$. scabiei were then compared with those of the reference strain S. scabiei 8722 (NC_013929.1), while sequences of S. acidiscabies LBUM 1476 was compared with the reference strain S. acidiscabies 84-104 (NZ_AHBF00000000.1). Sequences were then translated to determine the impact of mutations on protein sequences. The effect of amino acid substitutions were then analyzed using Protein Variation Effect Analyzer (PROVEAN) ${ }^{1}$ (Choi and Chan, 2015). Variants with a score equal to or below -2.5 were considered "deleterious," contrary to variants with a score above -2.5 which were considered as "neutral." The predicted secondary structures of tRNA were determined using tRNAscan$\mathrm{SE}^{2}$ (Lowe and Chan, 2016).

\footnotetext{
${ }^{1}$ http://provean.jcvi.org/seq_submit.php

${ }^{2}$ http://lowelab.ucsc.edu/tRNAscan-SE/index.html
} 


\section{RESULTS}

\section{General Characteristics of the Genome Sequences}

The genomes of 14 common scab-causing Streptomyces strains isolated from diseased tubers sampled in Canadian commercial potato production fields were sequenced, assembled, and annotated. Genomic features, including chromosome size, $\mathrm{G}+\mathrm{C} \%$ content, CDSs and contig numbers, are presented in Table 1. Briefly, the chromosome sequence sizes vary between 9.63 and $10.94 \mathrm{Mbp}$ (average 9.98) with a total number of CDSs ranging between 8373 and 9751 (average 8718) and a $\mathrm{G}+\mathrm{C} \%$ fluctuating between 70.6 and $71.6 \%$ (average 71.4). Each genome contains six rRNA operons (containing 5S, 16S, and $23 \mathrm{~S}$ rRNA) and between 70 and 75 tRNA genes.

\section{Phylogeny and Taxonomy}

In silico DNA-DNA hybridization and ANI calculations were performed to estimate genome similarity and taxonomical affiliation between the 14 Streptomyces strains under study. All Streptomyces strains except strain LBUM 1476 belong to the S. scabiei species with an average GGDC and ANI values of 95.68 and $99.56 \%$, respectively. LBUM 1476 was instead associated with $S$. acidiscabies with a GGDC of $99.2 \%$ and ANI of $99.97 \%$. For each of the 14 Streptomyces strains, the GGDC and ANI values yield similar results for species discrimination (Supplementary Table 2).

A phylogenomic tree of the 14 Streptomyces strains, alongside eight additional pathogenic Streptomyces spp. and an outgroup was constructed (Figure 1). This tree was based on the coregenome of these 23 strains, that corresponds to 1577 CDSs. This analysis showed consistent results with earlier observations using either ANI or GGDC values.

Four groups of S. scabiei could been distinguished according to an arbitrary threshold of GGDC > 98\% and ANI > 99.80\%. These groups were supported in the tree by $100 \%$ bootstrap values. The S. scabiei group I is the largest group comprising S. scabiei LBUM 1475, LBUM 1477, LBUM 1482, LBUM 1484, LBUM 1487, and LBUM 1488. All strains belonging to this group produce the melanin pigment contrary to those belonging to group II that include S. scabiei LBUM 1480, LBUM 1485, and LBUM 1486 (Hudec et al., 2021). Groups III and IV which also produced melanin, are the smallest groups and contain S. scabiei LBUM 1481/LBUM 1483 and LBUM 1478/LBUM 1479, respectively. Group IV is was the most distinct when compared to the other three groups.

\section{Analysis of a Potential Link Between the Phylogenetic Groups and Virulence}

To determine if a link exists between the phylogenetic groups identified and the virulence observed in a previous study (Hudec et al., 2021), we compared the S. scabiei group affiliation with the severity of symptoms observed (Table 2). Globally, strains belonging to the S. scabiei groups I and II display weak to intermediate virulence contrary to group IV, which contains intermediate to aggressive strains. Group III presents a higher virulence variability (Table 2 ). These results suggest the existence of distinct virulence factors or differential production of these factors between, and in some cases among the different S. scabiei groups.

\section{Characterization of the Pan-Genome}

To further characterize and compare the functional intra- and inter-species diversity, the pan-genome, which represents the entire gene repertory of the 14 Streptomyces under study, was analyzed (Figure 2). A total of 14,236 CDSs was recorded, of which almost half were poorly characterized (categories $\mathrm{S}$, $\mathrm{ND}$, and $\mathrm{X}$ ). $23 \%$ of the characterized CDSs were involved in metabolism (C, E, F, G, H, P, and Q categories), $19 \%$ in information storage processing (B, I, J, K, and L categories), and $13 \%$ in cellular processes and signaling (including the others COG categories). Moreover, among the 14,236 CDSs identified, 129 were found in VFDB (data not shown).

The $S$. acidiscabies isolate and the $13 \mathrm{~S}$. scabiei strains shared 2354 genes (i.e., core-genome) which principally encode essential function such as transcription (K; with $11.7 \%$ ) and amino acid metabolism and transport (E; 6.9\%). Only a small or no portion of the core-genome was associated with cell motility $(\mathrm{N} ; 0 \%)$, defense mechanisms $(\mathrm{V} ; 2 \%)$, and secondary metabolites biosynthesis (Q; 1.9\%). A higher prevalence of these gene categories was instead found to be group- or strain- specific. Moreover, $67 \%$ (85) of the VFs identified in this study were shared among the 14 strains (data not shown). The accessory genome which corresponds to all the genes present in two or more strains but not all contains a total of 9173 genes. S. acidiscabies and S. scabiei contain 1959 (including 15 VFs) and 1083 (21 VFs) species-specific genes, respectively. Only 6 VFs were found to be shared between at least two of the 14 strains, but were not group or species-specific (data not shown). These VFs were (with few exceptions) mostly present in groups II and IV and were involved in transcriptional regulation (3), stress responses (2), and the last one was a transposase.

The S. scabiei pan-genome (11,069 genes) included 3430 genes shared among the four S. scabiei groups. Among each S. scabiei group, more than $60 \%$ of genes were shared by all strains belonging to this group, displaying a high intragroup conservation. Each of these groups presents several group-specific genes (from 68 in group I to 228 in group III), mainly involved in replication, recombination and DNA repair categories $(\mathrm{L})$. The $\mathrm{N}, \mathrm{V}$, and $\mathrm{Q}$ categories potentially involved in pathogenesis and virulence displayed no differences in proportion between the four groups. Moreover, none of the VFs identified were $S$. scabiei group-specific (data not shown). Finally, a total of 750 unique genes were found among the 13 S. scabiei strains, and again, no match was found against the VFDB (data not shown).

\section{Comparative Analysis of the Pathogenicity Island}

To better understand the distinct patterns of virulence observed in a previous study using the same 14 Streptomyces strains (Hudec et al., 2021), the pathogenicity islands (PAI) containing several 
TABLE 2 | In silico analysis of the pathogenic determinants involved in pathogenicity and/or virulence of scab-causing Streptomyces spp., and their relative virulence and thaxtomin A production.

Phytotoxins

Phytohormones

Secreted proteins

Virulence $^{\mathrm{a}} *$ Thaxtomin A production $^{\mathrm{a}}$

Phylogenetic Species Strain Thaxtomin A Coronafacoyl Conca Desmethylm Borrelidin FD-891 Cytokinins Auxin Ethylene Nec1 TomA Scabin Suberinase

group namycin $A$ ensacarcin

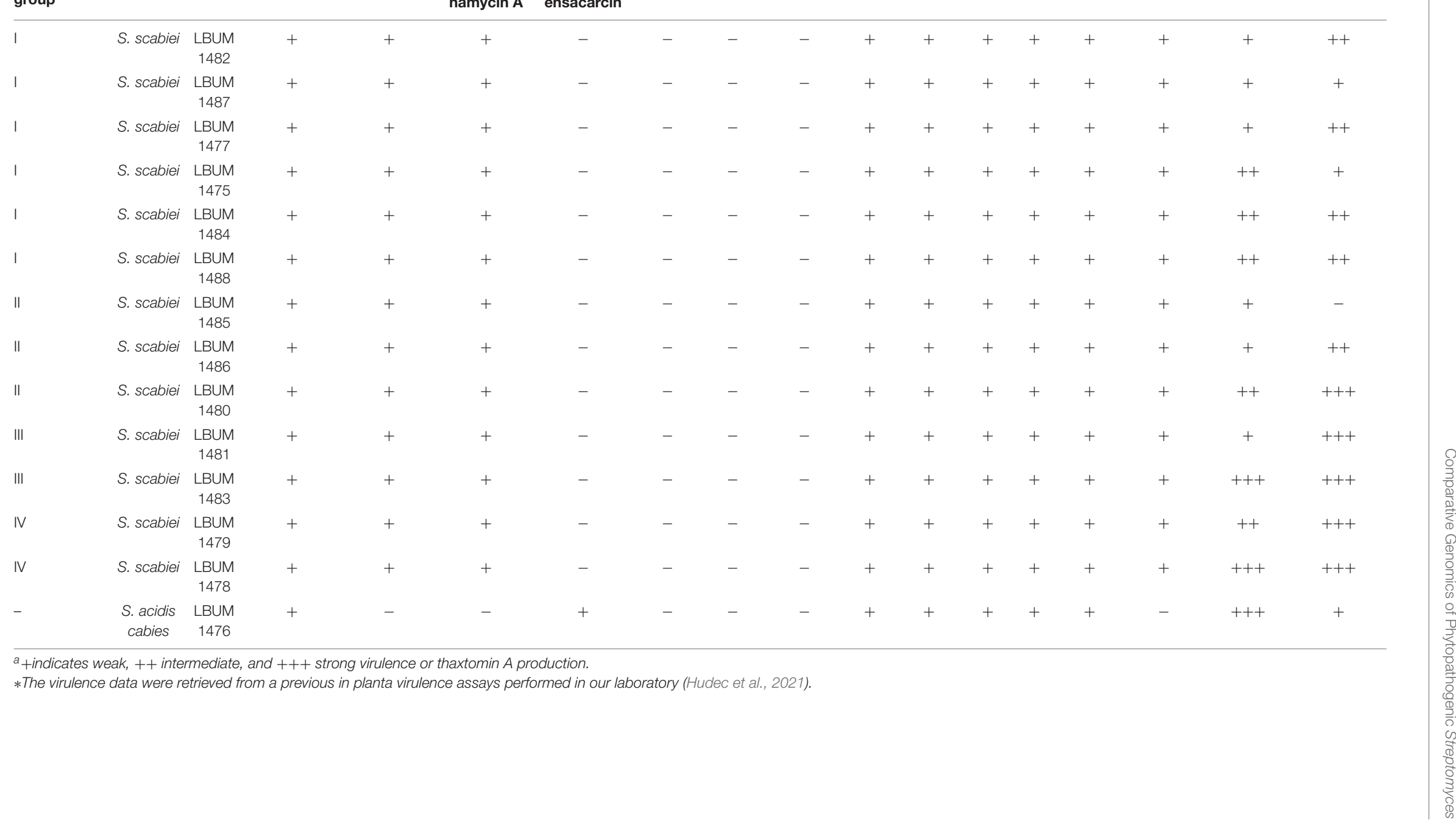


A

COG categories

Total number of CDSs

(J) Tranlsation, ribosomal structure and biogenesis (U) Intracellular trafficking, secretion, and vesicular transport

(F) Nucleotide metabolism and transport (H) Coenzyme metabolism and transpor

(C) Energy production and conversion

(P) Inorganic ion transport and metabolism

(E) Amino Acid metabolism and transport

(l) Lipid metabolism and transport

( $\mathrm{T})$ Signal transduction mechanisms

(K) Transcription

(O) Post-translational modification, protein turnover, chaperone functions

(D) Cell cycle control, cell division, chromosome partitioning

(M) Cell wall/membrane/envelop biogenesis

(G) Carbohydrate metabolism and transport

(V) Defense mechanisms

(S) Function unknown

(X) Assigned multiple $\mathrm{COG}$

(L) Replication, recombination and repair

(Q) Secondary metabolites biosynthesis, transport and catabolism

(ND) Not determined

(N) Cell motility

(B) Chromatin Structure and dynamics

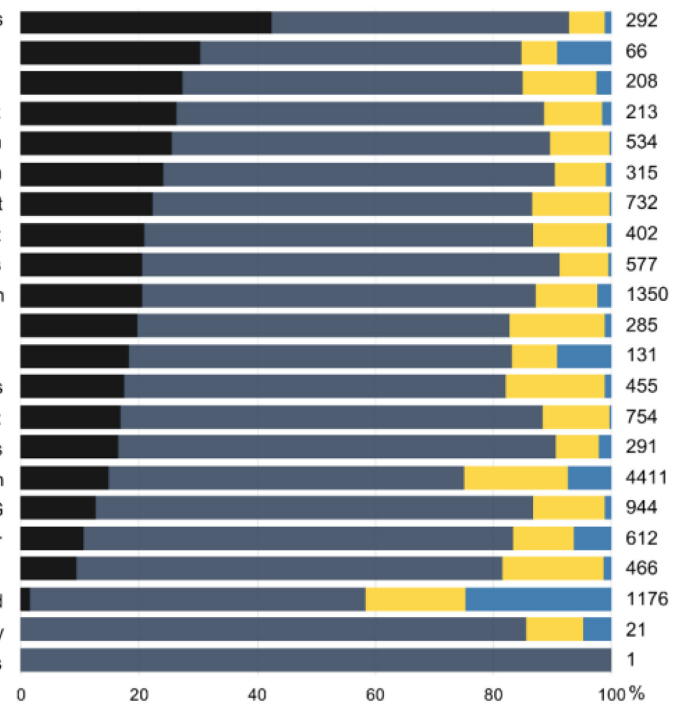

Core genome Accessory genome Singletons of S. acidiscabies Singletons of S. scabiei

B

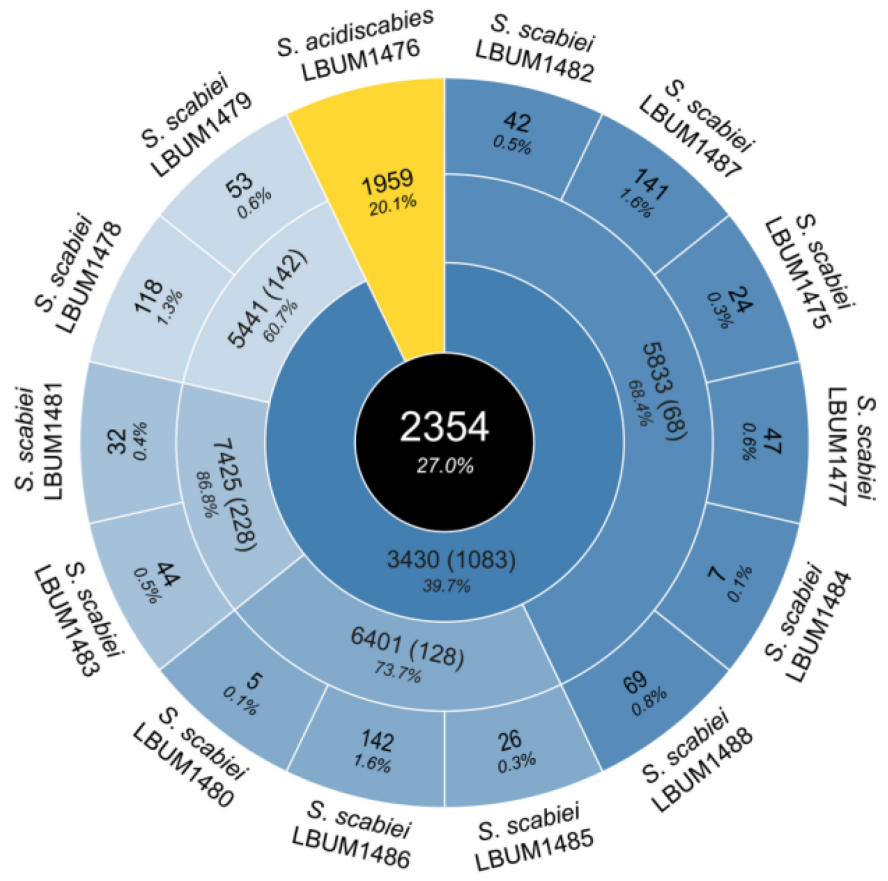

FIGURE 2 | Distribution of the orthologous CDSs from the pan-genome of the 14 scab-causing Streptomyces strains under study based on the COG categories (A) and their numbers belonging to the core-genome, group-specific, and strain specific genomes (B). In the histogram, bars are sorted by the proportion of CDS associated with the core-genome, the accessory genome and singletons (in S. acidiscabies and S. scabiel) in each functional category (A). The numbers presented in the circles from inside to outside: The black circle shows the core-genome of the 14 strains used in this study. The second circle shows the core-genome of each species - i.e., the 13 S. scabies (blue) and the only S. acidiscabies (yellow). The third circle compares the different Streptomyces scabiei core-genomes. Finally, the fourth and outermost circle represents the singletons (i.e., the CDSs that are strain-specific). The numbers in brackets indicate the number of CDSs that are species or group-specific (i.e., CDSs present in every strains from a species or a group but absent in all other strains). The percentages indicate the proportion of core genes (or singletons) compared to the average number of CDSs in the relevant set of strains (e.g., the core-genome of $2302 \mathrm{CDSs}$ found for the 14 strains from Streptomyces represent, in average, $26.4 \%$ of the total number of CDSs for each strain from this set) (B). 
pathogenic determinants were compared. Conservation of the PAI organization among each S. scabiei group was observed (Figure 3). More specifically, TR1 which contains the entire biosynthetic gene cluster for thaxtomin A production was highly conserved with $97.3 \%$ pairwise identity. However, TR2 was not found in S. scabiei group III and S. acidiscabies LBUM 1476.

The CR region displayed $66.7 \%$ pairwise identity, and two distinct organizations were observed: the classical one with $105 \mathrm{~kb}$ delimited by the $a t t L^{*}$ and att $\mathrm{R}^{*}$ sites, containing necl, $\operatorname{tom} A$ and several genes involved in the sugar catabolism; and a second one found only in S. scabiei group IV, containing a large insert of $165 \mathrm{~kb}$ (Figure 3). This insert was a concatenation of five fragments separated by diverse transposases. These fragments were found in all the strains belonging to S. scabiei group II, outside the $\mathrm{CR}$ region. Moreover, two distinct palindromic att $\mathrm{L}^{*}$ sites were distinguished. The TGTATGAA sequence was found in S. scabiei groups II and IV contrary to the TCTATGAA sequence found in the other S. scabiei groups and in S. acidiscabies LBUM 1476 (Figure 3). In the 14 strains, a degenerated version of intSt identified in S. turgidiscabies was found. MAFFT alignment of these sequences presented $94.3 \%$ of pairwise identity (data not shown).

\section{Production of Thaxtomin A}

Thaxtomin A is considered to be the main pathogenic determinant in scab-causing Streptomyces. In this context, its production by the 14 Streptomyces strains under study was quantified using a standardized assay to determine if differential production between the strains can explain their difference in virulence. Thus, thaxtomin A production and secretion was quantify by LC-MS. Results are presented in Figure 4. On average, S. acidiscabies LBUM 1476 produced $5.8 \mu \mathrm{g}$ of thaxtomin A per $\mathrm{ml}$ of supernatant. Except for strain LBUM 1485 , which did not produce any detectable amount of thaxtomin A, S. scabiei strains produced larger amounts of thaxtomin A than S. acidiscabies LBUM 1476. Indeed, concentrations ranging between 8.1 (S. scabiei LBUM 1487) and $44.8 \mu \mathrm{g} / \mathrm{ml}$ (LBUM 1479) are reported here (Figure 4A). Surprisingly, no correlation between the amount of thaxtomin A produced in vitro and the severity of symptoms observed in planta was found (Figure 4B; Hudec et al., 2021).

Moreover, similar production within group III (on average $32.3 \mu \mathrm{g} / \mathrm{ml})$ and IV $(43.8 \mu \mathrm{g} / \mathrm{ml})$ was observed. A higher heterogeneity was observed for the S. scabiei strains belonging to groups I and II, but, overall, these groups produced lower amounts of thaxtomin A than groups III and IV.

\section{Analysis of the Genetic Background Involved in the Production of Thaxtomin A}

The 14 Streptomyces strains under study produced different amounts of thaxtomin A. In order to elucidate the genetics differences involved in this differential production, the entire biosynthetic gene cluster involved in thaxtomin A production (including $t x t A, t x t B, t x t C, t x t D, t x t E$, and $t x t H$ genes) was compared. A MAFFT alignment of the concatenate CDSs was performed and showed $99.9 \%$ pairwise identity and $99.3 \%$ identical sites (data not shown). Conversion into protein sequences identified a total of 37 amino acid (AA) substitutions (Supplementary Table 3). Only one of these AA substitutions (a tyrosine at the position 166 substituted by a histidine; Y166H) was predicted to have an impact on the functionality of the nitric oxide synthase encoded by the $t x t D$ gene in S. scabiei LBUM 1475, LBUM 1481, and LBUM 1483 (Supplementary Table 3).

In addition, all the known genes involved in the regulation of thaxtomin A production were also compared $(t x t R, b l d C, b l d D$, bldG, bldH, CebE, CebF, CebG, MsiK, and CebR). None of the 12 AA substitutions found were predicted to impact the biological function of the corresponding proteins. Moreover, no nucleotide mutation was found on CebR binding sites among the strains. Finally, one S. scabiei strain (LBUM 1485) presented a mutation in bldA which is a tRNA Leu-tRNA ${ }^{U U A}$. In this case, its impact on the secondary structure was analyzed. This mutation was not found on any conservative loops (the anticodon, the $\mathrm{D}$ - and TYC loop). The base substitution (G6A) observed was on the acceptor stem and is predicted to disrupt the pairing with the corresponding nucleotide (C77) (Supplementary Figure 1).

\section{Comparative Presence/Absence and Protein Sequence Variations in Virulence Determinants Among the 14 Streptomyces spp.}

Although thaxtomin A is considered as the main virulence determinant in Streptomyces spp. causing CS, others phytotoxins, phytohormones and secreted proteins have been characterized as playing a role in the development and/or the severity of the disease. The $13 \mathrm{~S}$. scabiei displayed the same virulence genetic background including the presence of all the genes required for the biosynthesis and regulation of the phytotoxins thaxtomin A, CFA-Ile, and concanamycin A; the phytohormones auxin and ethylene; and the secreted proteins Nec1, TomA, scabin, and suberinase. S. acidiscabies LBUM 1476, for its part, presented a distinct pattern of virulence determinants. It does not harbor the genes involved in the biosynthesis of CFA-Ile, concanamycin A and suberinase, but it harbors those involved in desmethylmensacarcin biosynthesis. None of the Streptomyces strains under study has the genetic determinants required for the production of borrelidin, FD-891, or cytokinins (Table 2).

Since no distinct genetic virulence pattern seems to explain the varying level of virulence observed for the 14 Streptomyces strains, the potential impact of genetic mutations in the genes encoding the biosynthesis and regulation of these virulence determinants was analyzed (Supplementary Table 3). A total of 305 mutations which substitute the corresponding AA of the genes encoding the CFA-Ile, concanamycin A, auxin, ethylene, TomA, and suberinase was observed in S. scabiei strains. All of these mutations were group-specific. Indeed, all strains belonging to a given group presented the same mutation. No mutation in the genes encoding mensacarcin, or the $\mathrm{Necl}$ and scabin proteins was identified. Thus, among the mutations observed, only 35 were predicted to have an impact on the functionality of the corresponding proteins (Table 3). 29 of these were found in the concanamycin biosynthetic gene cluster and none 


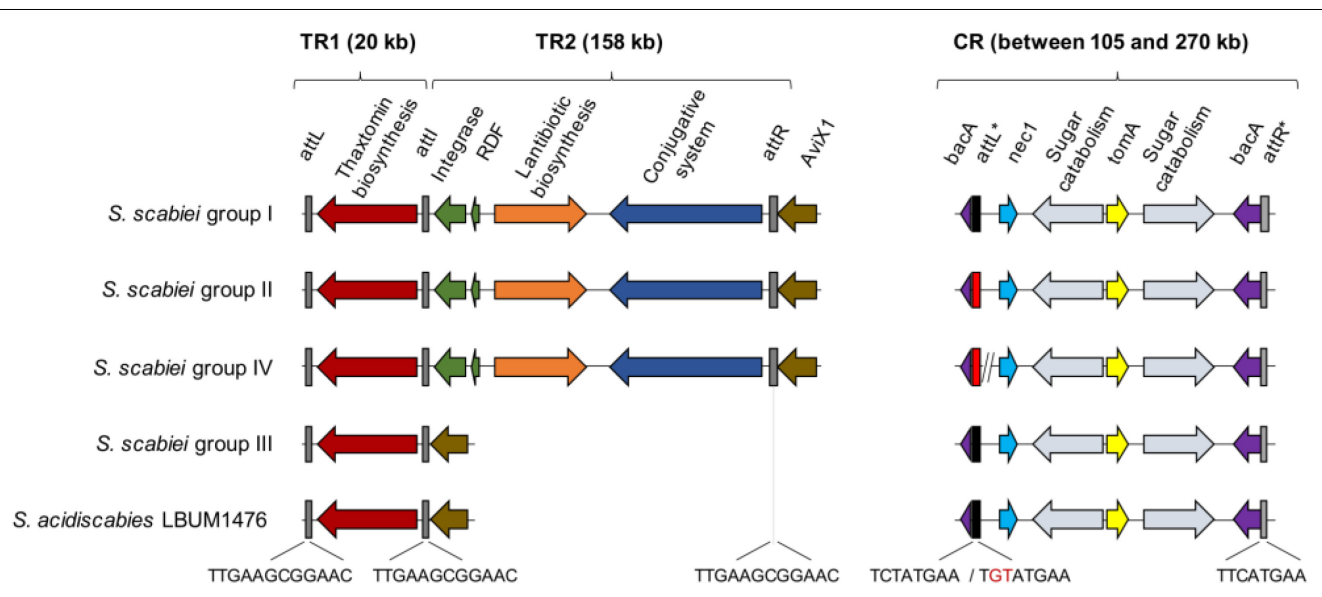

FIGURE 3 | Comparative analysis of the organization of the pathogenicity island between the fourteen scab-causing Streptomyces spp. used in this study. Each color corresponds to a gene or a cluster of genes homologous between the fourteen strains.

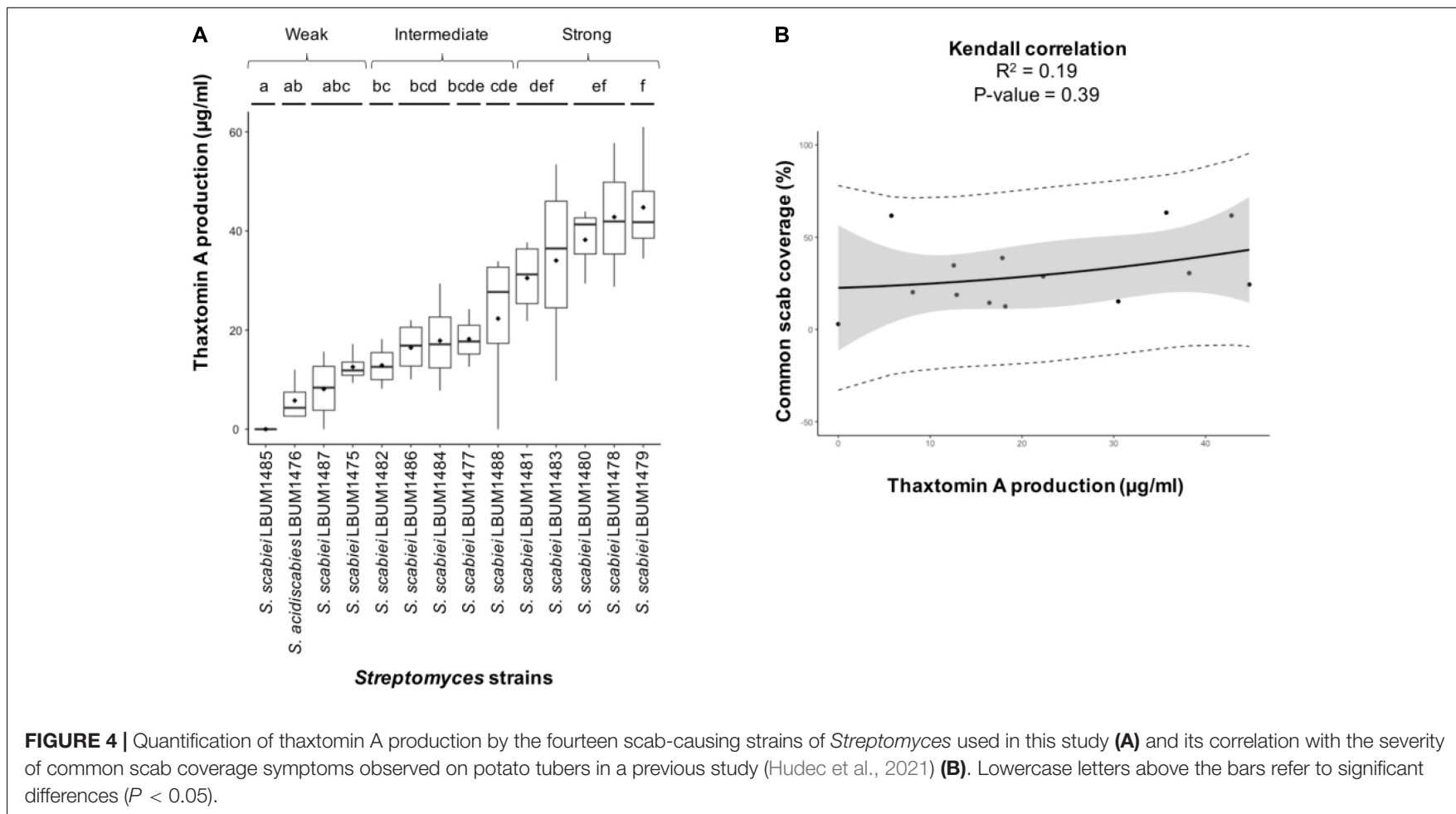

of them were found in the regulatory gene corresponding to SCAB_84101 in S. scabiei 87.22. Three mutations were found in the coronatine-like metabolite biosynthetic gene cluster, with one in the orf 1 potentially involved in the regulation of its production. Finally, two mutations were found in the iaaM gene (tryptophan monooxygenase enzyme) involved in the indole3 -acetamide (IAM) pathway and one in the gene encoding an ethylene forming enzyme (efe2).

The MAFFT alignment of the concatenated protein sequences showed $99.7 \%$ of pairwise identity. Moreover, all virulence determinants seem to have evolved in a group-specific way. Thus, contrary to the core-genome tree (Figure 1), the S. scabiei group
I seems to be more closely affiliated with group III, then with groups II and IV (Supplementary Figure 2).

\section{DISCUSSION}

In this study, we presented a comprehensive comparative genomic analysis of fourteen scab-causing Streptomyces strains (13 S. scabiei and one S. acidiscabies) with a focus on their PAI and VFs in order to better understand the origin of their distinct virulence patterns observed in a previous study (Hudec et al., 2021). 
The pan-genome analyses of these fourteen strains showed that the proportion of the core-genome range from 24.1 to $28.1 \%$ in each strain. With the lowest core-genome proportion (24.1\%) and the highest number of genes (9751), S. acidiscabies LBUM 1476 was the more divergent strain under study. Another study performed on the genome of five Streptomyces species had previously identified a core-genome varying between 33 and $45 \%$ in each species (Zhou et al., 2012). In our study, with only two distinct species, we would have expected the proportion of

TABLE 3 | Protein mutations encoded by genes involved in the production and/or regulation (bold) of pathogenicity/virulence factors in Streptomyces spp. used in this study.

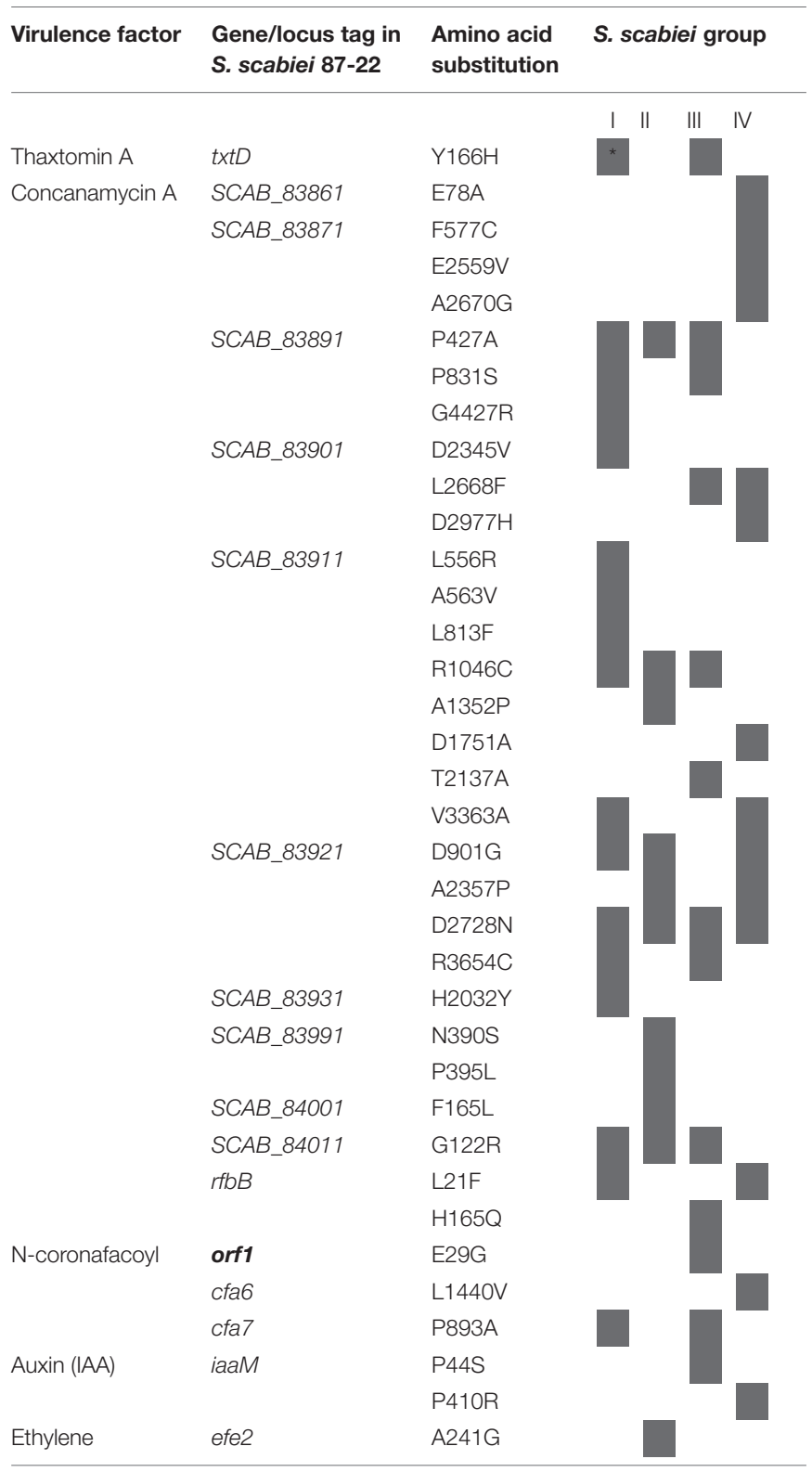

Sequences were compared to the reference strains S. scabiei 87-22 and mutations are indicated in gray.

*The mutation was only found in S. scabiei LBUM 1475. Bold refers to genes involved in regulation. common genes to be higher. Thus, this low proportion suggests a high heterogeneity between these fourteen strains, which was not only due to the presence of one S. acidiscabies strain. This is confirmed by the proportion of genes shared between the 13 S. scabiei strains, corresponding to $39.7 \%$ of their gene content. In comparison, another study performed on Streptomyces species had shown between 85 and $88 \%$ of genes shared among five Streptomyces pratensis strains (Doroghazi and Buckley, 2014). It is generally assumed that in a typical bacterial genome, a high proportion of the accessory genome is often associated with horizontal gene transfer (HGT) (Kim et al., 2015; Chater, 2016). Thus, the low proportion of core genes and the high proportion of accessory genes observed in S. scabiei species (62.2\%) suggests widespread HGT within this species, which may have contributed to the high heterogeneity. It is not surprising since it has already been demonstrated that Streptomyces spp. are able to perform HGT within and between species belonging to this genus (Doroghazi and Buckley, 2010). This highlights the existence of a high intra-species heterogeneity inside $S$. scabiei. In pathogenic bacterial strains, the accessory genes are often associated with virulence. Indeed, a loss in some of these genes is usually accompanied by a reduction in virulence (Medini et al., 2005). Here, no correlation between the virulence observed in a previous study and the size of the accessory genome determined in this study was found, suggesting that the varying virulence is more strain-specific than species-specific. Indeed, a study performed on several strains belonging to the Streptomyces albus species concluded that each stain harbors at least one strainspecific biosynthetic gene cluster (Seipke, 2015). In this study, a total of 750 S. scabiei strain-specific genes were identified. However, none of them was associated with the biosynthesis of any known virulence factor.

Despite this heterogeneity, the 13 S. scabiei strains used in this study displayed the same pathogenome, distinct from the one of $S$. acidiscabies LBUM 1476. Thus, despite the existence of common genes involved in the biosynthesis of several VFs, some of them were species-specific. The presence of the biosynthetic gene clusters involved in the biosynthesis of thaxtomin A, Nec1, or TomA in both species was not surprising because these proteins were classically found in Streptomyces spp. causing CS (Bignell et al., 2010). Moreover, the absence of the genes coding for the biosynthesis of FD-891, borrelidin, or the fas operon involved in the biosynthesis of cytokinins was also expected as these virulence factors have so far only been found in other Streptomyces species (notably in S. turgidiscabies) (Kers et al., 2004; Natsume et al., 2005, 2018; Cao et al., 2012). The presence of the biosynthetic gene clusters involved in CFA and concanamycin A production exclusively found in S. scabiei species was also expected (Natsume et al., 1996, 1998; Fyans et al., 2015, 2016; Bignell et al., 2018). However, until now the production of scabin, auxin and the presence of the gene encoding an ethyleneforming enzyme (efe) were only described in S. scabiei (Weingart et al., 2001; Bignell et al., 2010; Hsu, 2010; Legault et al., 2011). Here, homologs of the genes involved in their biosynthesis were also found in S. acidiscabies LBUM 1476. Moreover, it is also the first time that the genes coding for the biosynthesis of desmethylmensacarcin are found in S. acidiscabies. Indeed, 
among the Streptomyces spp. causing CS, this phytotoxin was only previously identified in S. niveiscabiei strain ST1015 (Lapaz et al., 2019). Its production by $S$. acidiscabies needs to be further investigated. Finally, among the enzymes which participates to the degradation of plant polymers to enhance thaxtomin A production, suberinase was only found in S. scabies (Komeil et al., 2013; Padilla-Reynaud et al., 2015). Its absence in S. acidiscabies may explain why this species produced less thaxtomin A than S. scabiei under the conditions used in this study.

The sequencing of thirteen S. scabiei genomes has allowed us to study in-depth the intra-species diversity. It is, however, not the first time that genetic heterogeneity inside S. scabiei is reported (St-Onge et al., 2008; Lapaz et al., 2017). Indeed, genotypic studies using DNA-DNA hybridization have previously distinguished at least three distinct groups within S. scabiei (Tashiro et al., 1990; Healy and Lambert, 1991; Paradis et al., 1994). Moreover, these S. scabiei groups were able to infect distinct crops as well as distinct potato cultivars with varying virulence (Keinath, 1989; Goyer and Beaulieu, 1997; Wanner, 2006). In our study, the analyses of the core-genome-based phylogeny and in silico genome similarity (ANI and is $\mathrm{DDH}$ ) has allowed us to distinguish four distinct $S$. scabiei genomic groups (groups I-IV).

Kers et al. (2004) identified the first PAI (PAISt, $674 \mathrm{~Kb}$ ) in Streptomyces turgidiscabies. Including the txt cluster, the fas operon, the nec1 and tomA genes, this PAISt was described as a combination of two modules of 105 and $569 \mathrm{~Kb}$ delimited by three att sites TTCATGAA (attL and attR at the extremity and attI between both modules). This PAI corresponds to an integrative and conjugative element (ICE) which may be entirely excised from the chromosome (by the recombinase IntSt and the putative excisionase XisSt) or partially excised (each individual module) and mobilized into a non-pathogenic strain through conjugation to be integrated into the receiving chromosome at a specific site (Kers et al., 2004; Huguet-Tapia et al., 2011, 2014).

In other Streptomyces spp. causing CS, this PAI was separated in two distinct regions by $4.9 \mathrm{Mb}$ : the toxicogenic and the colonization regions, TR and CR, respectively (Lerat et al., 2009). In these species, and notably in S. scabiei and S. acidiscabies, only remnants of intSt and distinct attL sequences bordering the CR were found (TGTATGAA for S. scabiei and TCTATGAA for S. acidiscabies) (Huguet-Tapia et al., 2014). In addition, in S. scabiei, the TR region was further divided into two sub-regions, the first one containing the txt cluster (TR1), and the second containing an antibiotic biosynthesis cluster, and potential ICE (TR2) (Chapleau et al., 2016). It has been demonstrated that for the strains containing TR2, each of the subregions and the full TR may be excised (Chapleau et al., 2016), but only TR and TR2 can be transferred and integrated specifically in a aviX1 site of a receiving strain (Zhang et al., 2016). Thus, TR1 is considering as a cis-mobilizable element (CIME) for which the TR2 (an ICE) is necessary to its mobilization (Zhang and Loria, 2017). Only the receiving strains having acquired the entire TR region develop a pathogenic phenotype and may participated to the emergence of new pathogenic species in an agricultural system (Kers et al., 2004; Zhang et al., 2016).
In our study, the four identified S. scabiei groups harbor four distinct organizations of the TR (TR1 and TR2) and CR regions. The TR1 region, which includes all the genes involved in the biosynthesis of thaxtomin A, was conserved among the fourteen Streptomyces spp. under study. Huguet-Tapia et al. (2016) previously demonstrated a possible acquisition of this region through lateral gene transfer that resulted in high conservation among Streptomyces sp. causing CS. However, contrary to what was previously reported by Zhang et al. (2016), the TR2 region was not conserved among the S. scabiei strains under study, since strains from group III do not harbor it. However, this observation supports data published by Chapleau et al. (2016) who identified pathogenic S. scabiei strains without a TR2 region. It was previously demonstrated that the TR1 region cannot be transferred to a saprophytic Streptomyces strain without the presence of the TR2 region (Zhang and Loria, 2017). Therefore, the absence of the TR2 region supports the assumption that strains belonging to the $S$. scabiei group III cannot contribute to the emergence of new pathogenic Streptomyces strains.

The CR region containing the necl and tom $A$ genes is also known to be conserved among pathogenic scab-causing Streptomyces strains (Zhang et al., 2016). Interestingly, the S. scabiei strains belonging to group IV presented a large insert, probably due to several transposition events inside the genome. Indeed, this sequence was found to be a combination of five genomic regions separated by several transposases. These five sequences were also found in the $S$. scabiei strains belonging to group II, suggesting that group IV might have evolved from group II. Moreover, this region is known to be delimited by an imperfect 8 bp palindromic att $L^{*}$ site presenting different nucleotide residues on the second and third base pairs depending on the species. However, in this study, S. scabiei strains from groups II to IV presented the attL* sequence identical to the one usually associated with $S$. acidiscabies species. It has already been suggested that the CR region may have been transferred in pathogenic Streptomyces spp. genomes via different horizontal gene transfer events than those associated with the TR region (Zhang et al., 2016). In this hypothesis, it would appear that this region may have been mobilized from $S$. acidiscabies to $S$. scabiei, but this will require further investigations.

Despite these four distinct organizations of the TR and CR regions, no correlation between the S. scabiei group affiliation of the 13 strains and virulence was found. Moreover, none of the VFs retrieved in this study were group specific. Despite the high heterogeneity found inside S. scabiei, no VFs identified at the species, group or strain level can explain the distinct pattern of virulence observed, suggesting the existence of other VFs presently unknown. This varying virulence may also perhaps be explained by the differential production of different VFs.

Indeed, it has already been demonstrated that a positive correlation exists between the amount of thaxtomin A produced and virulence (Kinkel et al., 1998; Loria et al., 2006). Despite the high conservation of the gene cluster involved in the biosynthesis of this phytotoxin, quantification of thaxtomin A produced in vitro showed a high variation in the amount produced between the strains under study. The S. scabiei strains from groups III to IV, which contain the most virulent strains, secreted the highest 
quantity of thaxtomin A. In contrast, group I, which is the less virulent group contains strains producing a weak or intermediate amount of thaxtomin A. Even if this production tends to be group-specific, no clear correlation between the quantity of thaxtomin A secreted and the virulence observed was found for the strains under study.

Moreover, among the different pathogenic Streptomyces spp. isolated throughout the world, a few reports have indicated that some pathogenic Streptomyces are capable of causing CS, while not producing thaxtomin A, usually caused by the absence of the TR region containing the thaxtomin A biosynthetic gene cluster (Wanner, 2004; Cao et al., 2012; Fyans et al., 2016; Lapaz et al., 2017). In this study, we instead identified a pathogenic strain (S. scabiei LBUM 1485) that has the entire thaxtomin A biosynthetic cluster but does not produce any detectable amount of thaxtomin $\mathrm{A}$, at least in the standardized in vitro assay used here. To our knowledge, this is the second report of a pathogenic Streptomyces strain containing the biosynthetic genes encoding thaxtomin A biosynthesis, but which does not produce this phytotoxin (Weisberg et al., 2021).

It has also been previously demonstrated that concanamycin A alone may induce common scab symptoms (Natsume et al., 2017). Therefore, the ability of S. scabiei strain LBUM 1485 to cause symptoms on potato tubers may be due to its capacity to produce concanamycin $\mathrm{A}$ as it harbors its entire biosynthetic gene cluster, however, this will require further investigation.

In order to understand why LBUM 1485 was not able to produce thaxtomin $\mathrm{A}$, at least under our experimental conditions, the entire thaxtomin A gene cluster was analyzed in detail. Surprisingly, across the 14 Streptomyces strains under study, no identifiable polymorphisms within the TR1 region, which may explain variations in virulence phenotypes, were found. The entire regulation system of thaxtomin A production was then compared. No mutation predicted to impact the structure or the functionality of the proteins were found neither in the $t x t R$ gene directly involved in the transcriptional regulation of the other $t x t$ genes (Joshi et al., 2007), the CebR cellulose utilization regulator, which is known to be the major regulator of virulence in S. scabies (Planckaert et al., 2018), nor the CebEFG/MsiK transmembrane transporter involved in the internalization of cellobiose and cellotriose (Jourdan et al., 2016). Moreover, homologs of all of these genes were found in S. acidiscabies LBUM 1476 suggesting that the differential production of thaxtomin A between the fourteen strains was not due to differential regulation by these systems. It has already been demonstrated that bldA and bldC mutants were unable to produce thaxtomin A (Bignell et al., 2014a). Indeed, bldA encodes the only leucyl-tRNA that can efficiently translate RNA containing a UUA codon, as found in the $t x t R$ sequence. A study performed on human tRNA has shown a low frequency of single nucleotide polymorphism (SNP) on the acceptor stem, D-stem, anticodon stem, and T-stem compared to flanking tRNA regions (Thornlow et al., 2018). Thus, the conservation of these regions seems to be particularly important to maintain the tRNA specificity and functionality. In our study, bldA was highly conserved among all S. scabiei strains, except for S. scabiei LBUM 1485, which presents a SNP on the acceptor stem (a $G$ at the position 6 was substituted by a $A$, which wobbles the G6:C78 link). It was further evidenced that the acceptor stem specifically confers tRNA identity. For example, the G3:U70 or the G1:A82/G2:C81 in Escherichia coli Ala-tRNA and Tyr-tRNA, respectively, confer the amino acid accepting specificity (aminoacylation) (Hooper et al., 1972; Celis et al., 1973; Hou and Schimmel, 1988). Considering this observation, this mutation was the best candidate identified in our study to explain the absence of thaxtomin A production by LBUM 1485. Complementary analyses will, however, need to be performed to confirm this observation.

Finally, despite the identification of a mutation in LBUM 1485 potentially explaining the absence of thaxtomin A production by this strain, none of the other comparative analyses performed in this study have been able to highlight with confidence why the fourteen strains display varying virulence. Therefore, using a similar approach as the one used for examining mutations in the thaxtomin A gene cluster, we investigated the potential impact of polymorphisms found on the biosynthetic clusters of all other known virulence determinants involved in the development of potato common scab. Despite the observation of numerous mutations predicted to impact the structure or functionality of the protein encoded, none could explain the virulence pattern observed.

In conclusion, in this study, a high inter-species genomic heterogeneity was found between S. scabiei and S. acidiscabies, with their respective species-specific pathogenome. Moreover, a high intra-species heterogeneity was also found inside S. scabiei with four distinct genomic groups. Despite this group specific pathogenic evolution, no virulence factors were determined to be group specific. Moreover, even among closely related strains belonging to the same group, a distinct pattern of virulence was often observed, suggesting the existence of strains-specific VFs. Indeed, no strong correlation between group affiliation and virulence was found. Similarly, no correlation between virulence and thaxtomin A production was observed, again supporting the existence of yet unknown VFs that are strain-specific. These results highlight the need for sequencing additional scab-causing Streptomyces spp., while in parallel characterizing their virulence. Larger-scale analyses will be required to make significant progress toward better understanding the genetic factors involved in virulence/pathogenicity. These progresses will undoubtedly be useful for developing targeted approaches for managing common scab of potato, a disease for which unfortunately no efficient control measure yet exist.

\section{DATA AVAILABILITY STATEMENT}

The datasets presented in this study can be found in online repositories. The names of the repository/repositories and accession number(s) can be found in the article/ Supplementary Material.

\section{AUTHOR CONTRIBUTIONS}

MF designed the research and supervised the work. $\mathrm{CH}$ performed the laboratory experiments and 
data analyses, and wrote the manuscript. $\mathrm{AB}$ helped with the bioinformatics analysis, including the design of some associated figures. RS-O helped with laboratory work. AN provided assistance with sequences submission. SL assisted with LC-MS use. JB assisted with the use of the EDGAR platform. All authors were involved in critical reading and reviewing the manuscript.

\section{FUNDING}

This work was supported by the New Brunswick Innovation Foundation and the Canadian Horticultural Council.

\section{REFERENCES}

Bignell, D. R. D., Cheng, Z., and Bown, L. (2018). The coronafacoyl phytotoxins: structure, biosynthesis, regulation and biological activities. Antonie Van Leeuwenhoek 111, 649-666. doi: 10.1007/s10482-017-1009-1

Bignell, D. R. D., Francis, I. M., Fyans, J. K., and Loria, R. (2014a). Thaxtomin A production and virulence are controlled by several bld gene global regulators in Streptomyces scabies. MPMI 27, 875-885. doi: 10.1094/MPMI-02-14-0037-R

Bignell, D. R. D., Fyans, J. K., and Cheng, Z. (2014b). Phytotoxins produced by plant pathogenic Streptomyces species. J. Appl. Microbiol. 116, 223-235. doi: 10.1111/jam.12369

Bignell, D. R. D., Huguet-Tapia, J. C., Joshi, M. V., Pettis, G. S., and Loria, R. (2010). What does it take to be a plant pathogen: genomic insights from Streptomyces species. Antonie Van Leeuwenhoek 98, 179-194. doi: 10.1007/s10482-010-9 429-1

Braun, S., Gevens, A., Charkowski, A., Allen, C., and Jansky, S. (2017). Potato common scab: a review of the causal pathogens, management practices, varietal resistance screening methods, and host resistance. Am. J. Potato Res. 94, 283-296. doi: 10.1007/s12230-017-9575-3

Cao, Z., Khodakaramian, G., Arakawa, K., and Kinashi, H. (2012). Isolation of borrelidin as a phytotoxic compound from a potato pathogenic Streptomyces strain. Biosci. Biotechnol. Biochem. 76, 353-357. doi: 10.1271/bbb.110799

Celis, J. E., Hooper, M. L., and Smith, J. D. (1973). Amino acid acceptor stem of E. coli suppressor tRNA tyr is a site of synthetase recognition. Nat. New Biol. 244, 261-264. doi: 10.1038/newbio244261a0

Chapleau, M., Guertin, J. F., Farrokhi, A., Lerat, S., Burrus, V., and Beaulieu, C. (2016). Identification of genetic and environmental factors stimulating excision from Streptomyces scabiei chromosome of the toxicogenic region responsible for pathogenicity: The toxicogenic region in S. scabiei strains. Mol. Plant Pathol. 17, 501-509. doi: 10.1111/mpp. 12296

Chater, K. F. (2016). Recent advances in understanding Streptomyces. F1000Res 5:2795. doi: 10.12688/f1000research.9534.1

Cheng, Z., Bown, L., Piercey, B., and Bignell, D. R. D. (2019). Positive and negative regulation of the virulence-associated coronafacoyl phytotoxin in the potato common scab pathogen Streptomyces scabies. MPMI 32, 1348-1359. doi: 10. 1094/MPMI-03-19-0070-R

Chin, C.-S., Alexander, D. H., Marks, P., Klammer, A. A., Drake, J., Heiner, C., et al. (2013). Nonhybrid, finished microbial genome assemblies from longread SMRT sequencing data. Nat. Methods 10, 563-569. doi: 10.1038/nmet h. 2474

Choi, Y., and Chan, A. P. (2015). PROVEAN web server: a tool to predict the functional effect of amino acid substitutions and indels. Bioinformatics 31, 2745-2747. doi: 10.1093/bioinformatics/btv195

De Mendiburu, F. (2019). Package 'agricolae' R Package, Version 1-2. Vienna: R Core Team.

Dieckmann, M. A., Beyvers, S., Nkouamedjo-Fankep, R. C., Hanel, P. H. G., Jelonek, L., Blom, et al. (2021). EDGAR 3.0: comparative genomics and phylogenomics on a scalable infrastructure. Nucleic Acids Res. 2021:gkab341. doi: 10.1093/nar/gkab341

Doroghazi, J. R., and Buckley, D. H. (2010). Widespread homologous recombination within and between Streptomyces species. ISME J. 4, 1136-1143. doi: $10.1038 /$ ismej.2010.45

\section{ACKNOWLEDGMENTS}

We would like to thank David Joly for his help with genomic analyses.

\section{SUPPLEMENTARY MATERIAL}

The Supplementary Material for this article can be found online at: https://www.frontiersin.org/articles/10.3389/fmicb. 2021.716522/full\#supplementary-material

Doroghazi, J. R., and Buckley, D. H. (2014). Intraspecies comparison of Streptomyces pratensis genomes reveals high levels of recombination and gene conservation between strains of disparate geographic origin. BMC Genomics 15:1-14. doi: 10.1186/1471-2164-15-970

Eason, J. R., Morris, R. O., and Jameson, P. E. (1996). The relationship between virulence and cytokinin production by Rhodococcus fascians (Tilford 1936) Goodfellow 1984. Plant Pathol. 45, 323-331. doi: 10.1046/j.1365-3059.1996. d01-130.x

Francis, I. M., Jourdan, S., Fanara, S., Loria, R., and Rigali, S. (2015). The cellobiose sensor CebR is the gatekeeper of Streptomyces scabies pathogenicity. mBio 6:14. doi: 10.1128/mBio.02018-14

Fyans, J. K., Altowairish, M. S., Li, Y., and Bignell, D. R. D. (2015). Characterization of the coronatine-like phytotoxins produced by the common scab pathogen Streptomyces scabies. MPMI 28, 443-454. doi: 10.1094/MPMI-09-14-0255-R

Fyans, J. K., Bown, L., and Bignell, D. R. D. (2016). Isolation and characterization of plant-pathogenic Streptomyces species associated with common scab-infected potato tubers in Newfoundland. Phytopathology 106, 123-131. doi: 10.1094/ PHYTO-05-15-0125-R

Goris, J., Konstantinidis, K. T., Klappenbach, J. A., Coenye, T., Vandamme, P., and Tiedje, J. M. (2007). DNA-DNA hybridization values and their relationship to whole-genome sequence similarities. Int. J. System. Evol. Microbiol. 57, 81-91. doi: 10.1099/ijs.0.64483-0

Goyer, C., and Beaulieu, C. (1997). Host range of Streptomycete strains causing common scab. Plant Dis. 81, 901-904. doi: 10.1094/PDIS.1997.81.8.901

Healy, F. G., and Lambert, D. H. (1991). Relationships among Streptomyces spp. causing potato scab. Int. J. System. Evol. Microbiol. 41, 479-482. doi: 10.1099/ 00207713-41-4-479

Hill, J., and Lazarovits, G. (2005). A mail survey of growers to estimate potato common scab prevalence and economic loss in Canada. Can. J. Plant Pathol. 27, 46-52. doi: 10.1080/07060660509507192

Hooper, M., Russell, R., and Smith, J. (1972). Mischarging in mutant tyrosine transfer RNAs. FEBS Lett. 22, 149-155. doi: 10.1016/0014-5793(72)80241-2

Hou, Y.-M., and Schimmel, P. (1988). A simple structural feature is a major determinant of the identity of a transfer RNA. Nature 333, 140-145. doi: 10. 1038/333140a0

Hsu, S.-Y. (2010). IAA production by Streptomyces scabies and its role in plant microbe interaction. Ph. D. thesis. Ithaca NY: Cornell University.

Hudec, C., Novinscak, A., and Filion, M. (2021). Diversity and virulence of Streptomyces spp. causing potato common scab in Prince Edward Island, Canada. Phytopathology 111, 617-626. doi: 10.1094/PHYTO-08-20-0339-R

Huerta-Cepas, J., Forslund, K., Coelho, L. P., Szklarczyk, D., Jensen, L. J., Von Mering, C., et al. (2017). Fast genome-wide functional annotation through orthology assignment by eggNOG-mapper. Mol. Biol. Evol. 34, 2115-2122. doi: $10.1093 / \mathrm{molbev} / \mathrm{msx} 148$

Huguet-Tapia, J. C., Badger, J. H., Loria, R., and Pettis, G. S. (2011). Streptomyces turgidiscabies Car8 contains a modular pathogenicity island that shares virulence genes with other actinobacterial plant pathogens. Plasmid 65, 118124. doi: $10.1016 /$ j.plasmid.2010.11.002

Huguet-Tapia, J. C., Bignell, D. R., and Loria, R. (2014). Characterization of the integration and modular excision of the integrative conjugative element PAISt in Streptomyces turgidiscabies Car8. PLoS One 9:e99345. doi: 10.1371/journal. pone.0099345 
Huguet-Tapia, J. C., Lefebure, T., Badger, J. H., Guan, D., Pettis, G. S., Stanhope, M. J., et al. (2016). Genome content and phylogenomics reveal both ancestral and lateral evolutionary pathways in plant-pathogenic Streptomyces species. Appl. Environ. microbiol. 82, 2146-2155. doi: 10.1128/AEM.03504-15

Jiang, G., Zhang, Y., Powell, M. M., Zhang, P., Zuo, R., Zhang, Y., et al. (2018). High-yield production of herbicidal thaxtomins and thaxtomin analogs in a nonpathogenic Streptomyces strain. Appl. Environ. Microbiol. 84, e164-e118. doi: 10.1128/AEM.00164-18

Johnson, E. G., Joshi, M. V., Gibson, D. M., and Loria, R. (2007). Cellooligosaccharides released from host plants induce pathogenicity in scab-causing Streptomyces species. Physiol. Mol. Plant Pathol. 71, 18-25. doi: 10.1016/j.pmpp. 2007.09.003

Joshi, M. V., and Loria, R. (2007). Streptomyces turgidiscabies possesses a functional cytokinin biosynthetic pathway and produces leafy galls. MPMI 20, 751-758. doi: 10.1094/MPMI-20-7-0751

Joshi, M. V., Bignell, D. R. D., Johnson, E. G., Sparks, J. P., Gibson, D. M., and Loria, R. (2007). The AraC/XylS regulator TxtR modulates thaxtomin biosynthesis and virulence in Streptomyces scabies. Mol. Microbiol. 66, 633-642. doi: 10.1111/j.1365-2958.2007.05942.x

Jourdan, S., Francis, I. M., Kim, M. J., Salazar, J. J. C., Planckaert, S., Frère, J.-M., et al. (2016). The CebE/MsiK transporter is a doorway to the cellooligosaccharide-mediated induction of Streptomyces scabies pathogenicity. Sci. Rep. 6, 1-12. doi: 10.1038/srep27144

Katoh, K., and Standley, D. M. (2013). MAFFT multiple sequence alignment software version 7: improvements in performance and usability. Mol. Biol. Evol. 30, 772-780. doi: 10.1093/molbev/mst010

Keinath, A. P. (1989). Population dynamics of Streptomyces scabies and other Actinomycetes as related to common scab of potato. Phytopathology 79, 681687. doi: 10.1094/Phyto-79-681

Kers, J. A., Cameron, K. D., Joshi, M. V., Bukhalid, R. A., Morello, J. E., Wach, M. J., et al. (2004). A large, mobile pathogenicity island confers plant pathogenicity on Streptomyces species: S. turgidiscabies pathogenicity island. Mol. Microbiol. 55, 1025-1033. doi: 10.1111/j.1365-2958.2004.04461.x

Kim, J.-N., Kim, Y., Jeong, Y., Roe, J.-H., Kim, B.-G., and Cho, B.-K. (2015). Comparative genomics reveals the core and accessory genomes of Streptomyces species. J. Microbiol. Biotechnol. 25, 1599-1605. doi: 10.4014/jmb.1504.04008

King, R. R., Lawrence, C. H., and Clark, M. C. (1991). Correlation of phytotoxin production with pathogenicity of Streptomyces scabies isolates from scab infected potato tubers. Am. Potato J. 68, 675-680. doi: 10.1007/BF02853743

Kinkel, L. L., Bowers, J. H., Shimizu, K., Neeno-Eckwall, E. C., and Schottel, J. L. (1998). Quantitative relationships among thaxtomin A production, potato scab severity, and fatty acid composition in Streptomyces. Can. J. Microbiol. 44, 768-776. doi: 10.1139/w98-061

Komeil, D., Simao-Beaunoir, A.-M., and Beaulieu, C. (2013). Detection of potential suberinase-encoding genes in Streptomyces scabiei strains and other actinobacteria. Can. J. Microbiol. 59, 294-303. doi: 10.1139/cjm-2012-0741

Lapaz, M. I., Huguet-Tapia, J. C., Siri, M. I., Verdier, E., Loria, R., and Pianzzola, M. J. (2017). Genotypic and phenotypic characterization of Streptomyces species causing potato common scab in Uruguay. Plant Dis. 101, 1362-1372. doi: 10.1094/PDIS-09-16-1348-RE

Lapaz, M. I., López, A., Huguet-Tapia, J. C., Pérez-Baldassari, M. F., Iglesias, C., Loria, R., et al. (2019). Isolation and structural characterization of a nondiketopiperazine phytotoxin from a potato pathogenic Streptomyces strain. Nat. Prod. Res. 33, 2951-2957. doi: 10.1080/14786419.2018.1511554

Lawrence, C. H., Clark, M. C., and King, R. R. (1990). Induction of common scab symptoms in aseptically cultured potato tubers by the vivotoxin, thaxtomin. Phytopathology 80, 606-608. doi: 10.1094/Phyto-80-606

Legault, G. S., Lerat, S., Nicolas, P., and Beaulieu, C. (2011). Tryptophan regulates thaxtomin A and indole-3-acetic acid production in Streptomyces scabiei and modifies its interactions with radish seedlings. Phytopathology 101, 1045-1051. doi: 10.1094/PHYTO-03-11-0064

Lerat, S., Simao-Beaunoir, A.-M., and Beaulieu, C. (2009). Genetic and physiological determinants of Streptomyces scabies pathogenicity. Mol. Plant Pathol. 10, 579-585. doi: 10.1111/j.1364-3703.2009.00561.x

Liu, B., Zheng, D., Jin, Q., Chen, L., and Yang, J. (2019). VFDB 2019: a comparative pathogenomic platform with an interactive web interface. Nucleic Acids Res. 47, D687-D692. doi: 10.1093/nar/gky1080
Loria, R., Kers, J., and Joshi, M. (2006). Evolution of plant pathogenicity in Streptomyces. Annu. Rev. Phytopathol. 44, 469-487. doi: 10.1146/annurev. phyto.44.032905.091147

Lowe, T. M., and Chan, P. P. (2016). tRNAscan-SE On-line: integrating search and context for analysis of transfer RNA genes. Nucleic Acids Res. 44, W54-W57. doi: 10.1093/nar/gkw413

Lyons, B., Ravulapalli, R., Lanoue, J., Lugo, M. R., Dutta, D., Carlin, S., et al. (2016). Scabin, a novel DNA-acting ADP-ribosyltransferase from Streptomyces scabies. J. Biol. Chem. 291, 11198-11215. doi: 10.1074/jbc.M115.707653

McCormick, J. R., and Flärdh, K. (2012). Signals and regulators that govern Streptomyces development. FEMS Microbiol. Rev. 36, 206-231. doi: 10.1111/j. 1574-6976.2011.00317.x

Medini, D., Donati, C., Tettelin, H., Masignani, V., and Rappuoli, R. (2005). The microbial pan-genome. Curr. Opin. Genet. Dev. 15, 589-594. doi: 10.1016/j.gde. 2005.09.006

Meier-Kolthoff, J. P., Auch, A. F., Klenk, H.-P., and Göker, M. (2013). Genome sequence-based species delimitation with confidence intervals and improved distance functions. BMC Bioinformatics 14:1-14. doi: 10.1186/1471-2105$14-60$

Natsume, M., Komiya, M., Koyanagi, F., Tashiro, N., Kawaide, H., and Abe, H. (2005). Phytotoxin produced by Streptomyces sp. causing potato russet scab in Japan. J. Gen. Plant Pathol. 71, 364-369. doi: 10.1007/s10327-005-0211-6

Natsume, M., Nagagata, A., Aittamaa, M., Okaniwa, N., Somervuo, P., Fiedler, H.P., et al. (2018). Phytotoxin produced by the netted scab pathogen, Streptomyces turgidiscabies strain 65, isolated in Sweden. J. Gen. Plant Pathol. 84, 108-117. doi: 10.1007/s10327-018-0765-8

Natsume, M., Ryu, R., and Abe, H. (1996). Production of phytotoxins, concanamycins A and B by Streptomyces spp. causing potato scab. Jap. J. Phytopathol. 62, 411-413. doi: 10.3186/jjphytopath.62.411

Natsume, M., Tashiro, N., Doi, A., Nishi, Y., and Kawaide, H. (2017). Effects of concanamycins produced by Streptomyces scabies on lesion type of common scab of potato. J. Gen. Plant Pathol. 83, 78-82. doi: 10.1007/s10327-017-0696-9

Natsume, M., Yamada, A., Tashiro, N., and Abe, H. (1998). Differential production of the phytotoxins thaxtomin A and concanamycins A and B by potato common scab-causing Streptomyces spp. Jap. J. Phytopathol. 64, 202-204. doi: 10.3186/ jjphytopath.64.202

Padilla-Reynaud, R., Simao-Beaunoir, A.-M., Lerat, S., Bernards, M. A., and Beaulieu, C. (2015). Suberin regulates the production of cellulolytic enzymes in Streptomyces scabiei, the causal agent of potato common scab. Microbes Environ. 30, 245-253. doi: 10.1264/jsme2.ME15034

Paradis, E., Goyer, C., Hodge, N. C., Hogue, R., Stall, R. E., and Beaulieu, C. (1994). Fatty acid and protein profiles of Streptomyces scabies strains isolated in Eastern Canada. Int. J. System. Bacteriol. 44, 561-564. doi: 10.1099/00207713-44-3-561

Park, D. H., Yu, Y. M., Kim, J. S., Cho, J. M., Hur, J. H., and Lim, C. K. (2003). Characterization of Streptomycetes causing potato common scab in Korea. Plant Dis. 87, 1290-1296. doi: 10.1094/PDIS.2003.87.1 1.1290

Planckaert, S., Jourdan, S., Francis, I. M., Deflandre, B., Rigali, S., and Devreese, B. (2018). Proteomic response to thaxtomin phytotoxin elicitor cellobiose and to deletion of cellulose utilization regulator CebR in Streptomyces scabies. J. Proteome Res. 17, 3837-3852. doi: 10.1021/acs.jproteome.8b00528

Richter, M., and Rosselló-Móra, R. (2009). Shifting the genomic gold standard for the prokaryotic species definition. PNAS 106, 19126-19131. doi: 10.1073/pnas. 0906412106

Rodriguez-R, L. M., and Konstantinidis, K. T. (2016). The enveomics collection: a toolbox for specialized analyses of microbial genomes and metagenomes. PeerJ Prepr. 4:e1900v1. doi: 10.7287/peerj.preprints.1900v1

Saitou, N., and Nei, M. (1987). The neighbor-joining method: a new method for reconstructing phylogenetic trees. Mol. Biol. Evol. 4, 406-425. doi: 10.1093/ oxfordjournals.molbev.a040454

Seipke, R. F. (2015). Strain-level diversity of secondary metabolism in Streptomyces albus. PLoS One 10:e0116457. doi: 10.1371/journal.pone.0116457

Statistics Canada (2021). Table 32-10-0358-01 Area, production and farm value of potatoes. Ottawa: Statistics Canada.

Stevenson, W. R., Loria, R., and Franc, G. D. (2001). Compendium of potato diseases, ed. D. P. Weingartner (Minnesota, MN: The American Phytopathological Society). 
St-Onge, R., Goyer, C., Coffin, R., and Filion, M. (2008). Genetic diversity of Streptomyces spp. causing common scab of potato in eastern Canada. Syst. Appl. Microbiol. 31, 474-484. doi: 10.1016/j.syapm.2008.09.002

Tashiro, N., Miyashita, K., and Suzui, T. (1990). Taxonomic studies on the Streptomyces species, isolated as causal organisms of potato common scab. Jap. J. Phytopathol. 56, 73-82. doi: 10.3186/jjphytopath.56.73

Tatusov, R. L., Galperin, M. Y., Natale, D. A., and Koonin, E. V. (2000). The COG database: a tool for genome-scale analysis of protein functions and evolution. Nucleic Acids Res. 28, 33-36. doi: 10.1093/nar/28.1.33

Tatusova, T., DiCuccio, M., Badretdin, A., Chetvernin, V., Nawrocki, E. P., Zaslavsky, L., et al. (2016). NCBI prokaryotic genome annotation pipeline. Nucleic Acids Res. 44, 6614-6624. doi: 10.1093/nar/gkw569

Thornlow, B. P., Hough, J., Roger, J. M., Gong, H., Lowe, T. M., and Corbett-Detig, R. B. (2018). Transfer RNA genes experience exceptionally elevated mutation rates. PNAS 115, 8996-9001. doi: 10.1073/pnas.1801240115

Wanner, L. A. (2004). Field isolates of Streptomyces differ in pathogenicity and virulence on radish. Plant Dis. 88, 785-796. doi: 10.1094/PDIS.2004.88.8.785

Wanner, L. A. (2006). A survey of genetic variation in Streptomyces isolates causing potato common scab in the United States. Phytopathology 96, 1363-1371. doi: 10.1094/PHYTO-96-1363

Weingart, H., Ullrich, H., Geider, K., and Völksch, B. (2001). The role of ethylene production in virulence of Pseudomonas syringae pvs. glycinea and phaseolicola. Phytopathology 91, 511-518. doi: 10.1094/PHYTO.2001.91.5.511

Weisberg, A. J., Kramer, C. G., Kotha, R. R., Luthria, D. L., Chang, J., and Clarke, C. R. (2021). A novel species-level group of Streptomyces exhibits variation in phytopathogenicity despite conservation of virulence loci. MPMI 34, 39-48. doi: 10.1094/MPMI-06-20-0164-R
Zhang, Y., and Loria, R. (2017). Emergence of novel pathogenic Streptomyces species by site-specific accretion and cis-mobilization of pathogenicity islands. MPMI 30, 72-82. doi: 10.1094/MPMI-09-16-0190-R

Zhang, Y., Bignell, D. R. D., Zuo, R., Fan, Q., Huguet-Tapia, J. C., Ding, Y., et al. (2016). Promiscuous pathogenicity islands and phylogeny of pathogenic Streptomyces spp. MPMI 29, 640-650. doi: 10.1094/MPMI-04-16-0068-R

Zhou, Z., Gu, J., Li, Y.-Q., and Wang, Y. (2012). Genome plasticity and systems evolution in Streptomyces. BMC Bioinformatics 13:1-17. doi: 10.1186/14712105-13-S10-S8

Conflict of Interest: The authors declare that the research was conducted in the absence of any commercial or financial relationships that could be construed as a potential conflict of interest.

Publisher's Note: All claims expressed in this article are solely those of the authors and do not necessarily represent those of their affiliated organizations, or those of the publisher, the editors and the reviewers. Any product that may be evaluated in this article, or claim that may be made by its manufacturer, is not guaranteed or endorsed by the publisher.

Copyright (c) 2021 Hudec, Biessy, Novinscak, St-Onge, Lamarre, Blom and Filion. This is an open-access article distributed under the terms of the Creative Commons Attribution License (CC BY). The use, distribution or reproduction in other forums is permitted, provided the original author(s) and the copyright owner(s) are credited and that the original publication in this journal is cited, in accordance with accepted academic practice. No use, distribution or reproduction is permitted which does not comply with these terms. 